\title{
Circular RNA mediates cardiomyocyte death via miRNA-dependent upregulation of MTP18 expression
}

\author{
Kun Wang ${ }^{1,4}$, Tian-Yi Gann ${ }^{2,4}$, Na Li ${ }^{3}$, Cui-Yun Liu', Lu-Yu Zhou, Jin-Ning Gao', Chao Chen ${ }^{1}$, Kao-Wen Yan ${ }^{1}$, Murugavel Ponnusamy ${ }^{1}$, \\ Yu-Hui Zhang ${ }^{\star 2}$ and Pei-Feng Li*,1
}

Circular RNAs (circRNAs) have important roles in several cellular processes. No study has established the pathophysiological role for circRNAs in the heart. Here, we show that a circRNA (mitochondrial fission and apoptosis-related circRNA (MFACR)) regulates mitochondrial fission and apoptosis in the heart by directly targeting and downregulating miR-652-3p; this in turn blocks mitochondrial fission and cardiomyocyte cell death by suppressing MTP18 translation. MTP18 deficiency reduces mitochondrial fission and suppresses cardiomyocyte apoptosis and MI. miR-652-3p directly downregulates MTP18 and attenuates mitochondrial fission, cardiomyocyte apoptosis, and MI in vitro and in vivo. MFACR directly sequesters miR-652-3p in the cytoplasm and inhibits its activity. MFACR knockdown in cardiomyocytes and mice attenuates mitochondrial fission and Ml. Our results reveal a crucial role for circRNA in regulating mitochondrial dynamics and apoptosis in the heart; as such, circRNAs may serve as a potential therapeutic avenue for cardiovascular diseases.

Cell Death and Differentiation (2017) 24, 1111-1120; doi:10.1038/cdd.2017.61; published online 12 May 2017

Circular RNAs (circRNAs) are an emerging class of noncoding RNAs that form covalently closed continuous loops. ${ }^{1,2}$ Researchers have found that numerous circRNAs are expressed in humans, mice, plants, and Drosophila, ${ }^{3-5}$ and exhibit spatial and temporal-specific expression. ${ }^{6}$ CircRNAs have been reported to have important roles in diseases of the nervous system and cancer, including colorectal cancer and pancreatic ductal adenocarcinoma. ${ }^{7-10}$ As such, circRNAs can serve as new diagnostic and treatment strategies for disease. CircRNAs, such as CDR1as/ciRS-7 and sex-determining region $Y$ (Sry), also act as a molecular sponge for miRNAs and function as post-transcriptional regulators by reducing miRNA expression. ${ }^{2,11}$ There is also a difference between the ratio of circular to linear RNA isoforms in normal tissues and that in tumor samples, which suggests that circRNAs can serve as diagnostic or predictive biomarkers of human diseases. ${ }^{8}$ However, the function of circRNAs in cardiomyocytes has remained unknown. Our study reveals - for the first time - that circRNA functions to regulate mitochondrial dynamics, cardiomyocyte apoptosis, and myocardial infarction (MI).

There are large amounts of mitochondria in the heart to provide enough energy to sustain the normal function of cardiomyocytes. An increasing number of studies have indicated that mitochondrial fission dysfunction occurs in many cardiac diseases, such as MI and heart failure. MTP18 is a nuclear-encoded mitochondrial membrane protein that has been reported to contribute to mitochondrial fission in mammalian cells. ${ }^{12,13}$ However, the exact function of MTP18 in heart diseases involving abnormal mitochondrial fission has rarely been studied.

miRNAs are noncoding RNAs that are 19-24 nucleotides long. They negatively regulate gene expression by inhibiting mRNA translation or increasing mRNA degradation. miR-652 has been identified in several tissues and exerts important functions. miR-652 has a potential effect on mediating the fibrogenic and inflammatory processes in the pathogenesis of liver cirrhosis. ${ }^{14}$ miR-652 may act as a suitable biomarker for distinguishing malignant pleural mesothelioma from reactive mesothelial proliferations. ${ }^{15}$ miR-652 has been reported to be a factor that mainly functions in myeloid regulation. ${ }^{16}$ In addition, miR-652 can suppress the epithelial-mesenchymal transition of pancreatic cancer cells in acidic microenvironment conditions by targeting ZEB $1 .{ }^{17}$ miR-652-3p promotes non-small-cell lung cancer proliferation and metastasis by directly targeting the lethal giant larvae $1 .{ }^{18}$ However, whether miR-652 participates in pathophysiological regulation in the heart remains unknown. It is also unclear whether miR-652 can target MTP18 to mediate mitochondrial network.

The aim of this study was to explore the role and potential underlying mechanism of circRNA (mitochondrial fission and apoptosis-related circRNA (MFACR)) and miR-652-3p in cardiac apoptosis and ischemia/reperfusion (I/R) injury. Here, we show that MFACR mediates cardiomyocyte apoptosis and MI by targeting miR-652-3p-dependent MTP18 activation and consequent mitochondrial fission. Our findings reveal a novel role for circRNA in regulating mitochondrial dynamics and

\footnotetext{
${ }^{1}$ Institute for Translational Medicine, College of Medicine, Qingdao University, Qingdao 266021, China; ${ }^{2}$ State Key Laboratory of Cardiovascular Disease, Heart Failure Center, Fuwai Hospital, National Center for Cardiovascular Diseases, Chinese Academy of Medical Sciences, Peking Union Medical College, Beijing 100037, China and ${ }^{3}$ State Key Laboratory of Biomembrane and Membrane Biotechnology, Institute of Zoology, Chinese Academy of Sciences, Beijing 100101, China

${ }^{*}$ Corresponding author: Y-H Zhang, State Key Laboratory of Cardiovascular Disease, Heart Failure Center, Fuwai Hospital, National Center for Cardiovascular Diseases, Chinese Academy of Medical Sciences, Peking Union Medical College, Beijing 100037, China. E-mail: zhangyhfw@ 163.com

or P-F Li, Institute for Translational Medicine, College of Medicine, Qingdao University, DengZhou Road, Qingdao 266021, China. Tel: +86 053282991039 ; Fax: +86 0532 83801449; E-mail: peifengliqd@ 163.com

${ }^{4}$ These authors contributed equally to this work.

Received 05.12.16; revised 15.3.17; accepted 31.3.17; Edited by RA Knight; published online 12.5.2017
} 
a

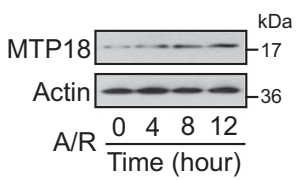

b
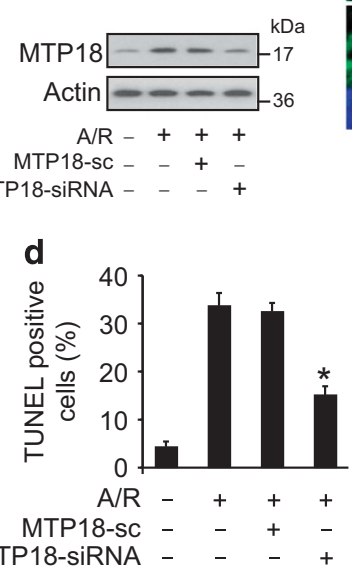

g

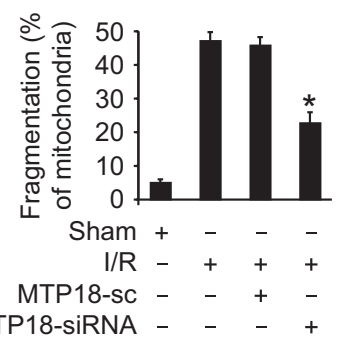

C
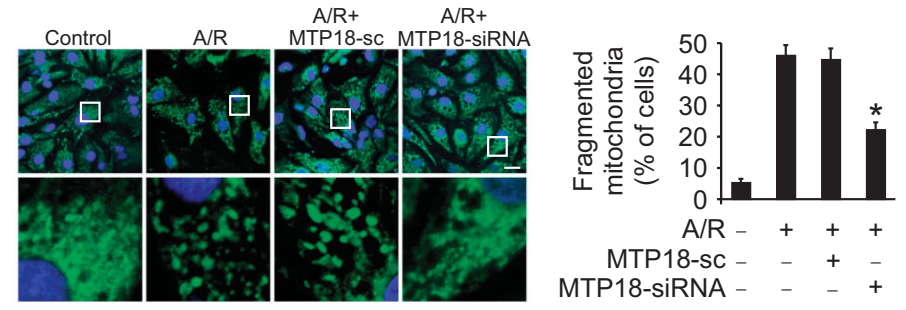

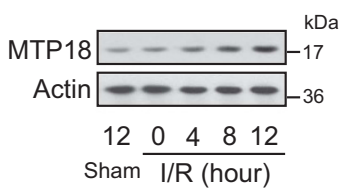

f

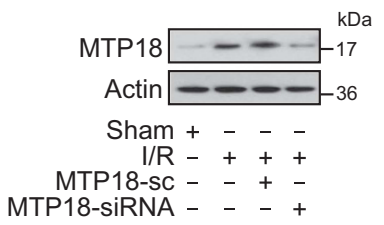

$\mathbf{h}$

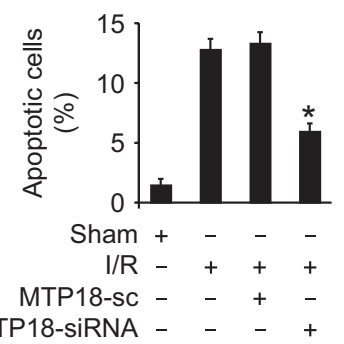

i
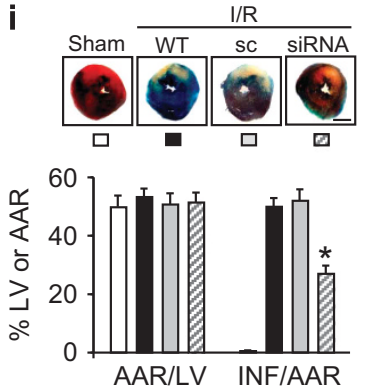

Figure 1 MTP18 regulates mitochondrial fission and apoptosis in vitro and in vivo. (a) A/R induces an increase in MTP18 levels. Cardiomyocytes were treated with A/R for the indicated times. MTP18 levels were analyzed by immunoblot. (b) Knockdown of MTP18 attenuates the A/R-induced increased of MTP18 levels. Cardiomyocytes were infected with adenoviral constructs of MTP18-siRNA or its scrambled form (sc) and then exposed to A/R. MTP18 levels were analyzed by immunoblot. (c) Knockdown of MTP18 attenuates mitochondrial fission induced by A/R treatment. Cardiomyocytes were infected with adenoviruses harboring MTP18-siRNA or its Sc form and treated with A/R. Mitochondria were stained by MitoTracker Red and the nuclei were visualized by 4',6-diamidino-2-phenylindole (DAPI) (left panel). Bar $=20 \mu \mathrm{m}$. The cells with fragmented mitochondria were counted (right panel). ${ }^{*} P<0.05$ versus A/R alone. (d) Knockdown of MTP18 reduces A/R-induced apoptosis. Cardiomyocytes were infected with adenoviruses harboring MTP18-siRNA or its sc form and then were subjected to A/R. TUNEL was used to analyze apoptotic cells. TUNEL-positive cells were counted. ${ }^{*} P<0.05$ versus A/R alone. (e) MTP18 is upregulated in response to I/R. Mice were subjected to various durations of ischemia, and MTP18 levels were detected by immunoblot. (f) MTP18-siRNA can efficiently knockdown MTP18 in the animal model. After intracoronary delivery of adenoviruses harboring MTP18-siRNA or the sc form, the mice were subjected to I/R injury. MTP18 levels were detected by immunoblot. ( $\mathbf{g}$ and $\mathbf{h}$ ) Knockdown of MTP18 attenuates I/R-induced mitochondrial fission and apoptosis. After intracoronary delivery of adenoviruses harboring MTP18-siRNA or the sc form, the mice were subjected to $45 \mathrm{~min}$ ischemia, followed by $3 \mathrm{~h}$ reperfusion. Mitochondrial fission was analyzed (g). TUNEL assay was used to detect apoptotic cells (h). ${ }^{*} P<0.05$ versus I/R alone. (i) Knockdown of MTP18 decreases Ml sizes in response to l/R. Mice were treated as described for (f), and infarct sizes were calculated. The upper panels are myocardial representative photos of midventricular myocardial slices. Bar $=2 \mathrm{~mm}$. ${ }^{*} P<0.05 \mathrm{versus} \mathrm{l} / \mathrm{R}$ alone

cardiomyocyte apoptosis, and the MFACR/miR-652-3p/ MTP18 axis as a regulator of apoptosis in the heart will be a potential therapeutic target for the treatment of cardiovascular diseases.

\section{Results}

MTP18 regulates mitochondrial fission and apoptosis in cardiomyocytes. Severe MI causes a condition with an absolute deficit in oxygen supply and provokes cardiomyocyte death, which consequently leads to cardiac dysfunction. To investigate the involvement of MTP18 in oxygen deprivation-induced apoptotic cell death in cardiomyocytes, cultured primary cardiomyocytes were subjected to anoxia/ reoxygenation $(A / R)$. The level of MTP18 was significantly elevated (Figure 1a) in cardiomyocytes in the A/R condition, which was accompanied by a significant increase in mitochondrial fission (Supplementary Figure 1a) and apoptosis (Supplementary Figure 1b). Next, we used a more specific siRNA of MTP18 to examine the influence of MTP18 in $A / R$-induced cardiomyocyte death. As shown in Figure 1b, the knockdown of MTP18 efficiently attenuated the A/Rinduced elevation in the MTP18 level in cardiomyocytes (Figure 1b). The mitochondrial morphology analysis showed that the suppression of MTP18 significantly reduced the number of cells with fragmented mitochondria (Figure 1c). In addition, the knockdown of MTP18 attenuated A/R-induced apoptosis, which was shown by a significant reduction in caspase-3 activity (Supplementary Figure 1c) and a 
decrease in the number of terminal deoxyribonucleotidyl transferase-mediated dUTP nick end labeling (TUNEL)positive cells (Figure 1d). These results indicate that MTP18 participates in the mitochondrial fission-mediated apoptotic cell death of cardiomyocytes during oxygen deprivation stress.

Next, we used a mouse I/R injury model of MI to investigate the contribution of MTP18 to the pathogenesis of MI in vivo. In the heart of mice with I/R-induced $\mathrm{MI}$, the expression of MTP18 was markedly increased compared with that in the control heart (Figure 1e), which indicates that MTP18 is associated with the development of MI. To define the role of MTP18 in the pathogenesis of MI, we administered MTP18siRNA to mice before the induction of I/R injury (Figure 1f). Interestingly, the delivery of MTP18-siRNA resulted in a significant reduction in $\mathrm{l} / \mathrm{R}$ injury-induced mitochondrial fragmentation (Figure 1g) and apoptosis (Figure 1h). The blockage of the elevation in MTP18 using siRNA significantly reduced the I/R-induced cardiac infarct size (Figure 1i). The echocardiography result showed that I/R-injury-induced abnormalities in cardiac function were ameliorated by silencing of MTP18 (Supplementary Figures 1d-g). Taken together, these data suggest that MTP18 mediates mitochondrial fission and apoptotic cell death in the heart.

miR-652-3p regulates MTP18 expression. Emerging evidence has revealed that miRNAs has a vital role in regulating mitochondrial dynamics as well as cardiomyocyte cell death by directly or indirectly targeting signaling molecules associated with these processes. To elucidate whether miRNA(s) regulate the expression of MTP18 to control mitochondrial fission, we first screened the expression levels of some known miRNAs in cultured cardiomyocytes that have been reported for their impact on many pathological and physiological processes. Among them, the miR-652-3p level was significantly downregulated in cardiomyocytes under A/R (Figure 2a). The levels of other miRNAs remained unchanged (data not shown). The bioinformatic analysis using the RNAhybrid program found that the 3'-UTR of MTP18 has a potential binding site for miR-652$3 p$ (Figure $2 b$ ), which prompted us to focus on the influence of miR-652-3p on the changes in the level of MTP18. Interestingly, the expression of miR-652-3p did not affect the level of MTP18 mRNA (Supplementary Figure 2a). In contrast, the suppression of miR-652-3p using an antagomir increased the level of MTP18 protein (Figure 2c). In addition, the forced expression of miR-652-3p attenuated the A/R-induced increase in the MTP18 protein level in cardiomyocytes (Figure $2 d$ ). These results indicate that miR-652-3p regulates MTP18 expression at the translational level without affecting its mRNA expression.

Next, we explored the inhibitory mechanism of the translation of MTP18 by miR-652-3p using a luciferase assay system. The results of the luciferase assay showed that the translation of wild-type MTP18 was inhibited by miR-652-3p as indicated by the reduced luciferase signal (Figure 2f). However, translational activity was unaffected by miR-652-3p when mutations were introduced in the MTP18 3'-UTR (Figures $2 \mathrm{e}$ and f). These results indicate that miR-652-3p targets the $3^{\prime}$-UTR of MTP18 to prevent its translation. To confirm this result, miR-652-3p was co-transfected with wild-type MTP18 or mutant MTP18 into cells, and the protein level of MTP18 was assessed. The level of MTP18 was relatively low in cells with wild-type MTP18 and miR-652-3p (Figure 2g). However, the MTP18 expression level was not affected in cells transfected with MTP18 with mutations in the miR-652-3p binding site in the $3^{\prime}$-UTR (Figure $2 \mathrm{~h}$ ). Collectively, these findings indicate that MTP18 is a downstream target of miR-652-3p.

miR-652-3p suppresses mitochondrial fission and apoptosis. Next, we elucidated the functional role of miR-652-3p in mitochondrial fission and apoptosis in cardiomyocytes. In cultured cardiomyocytes, the forced expression of miR-652$3 p$ efficiently replenished its level in cells in the A/R condition (Supplementary Figure 2b), and this increase in miR-652-3p attenuated the A/R-induced mitochondrial fission (Figure 3a) and apoptosis (Figure 3b). These data indicate that miR-652$3 p$ acts as a negative regulator and inhibits mitochondrial fission and apoptosis in cardiomyocytes. Similarly, the level of miR-652-3p was decreased in the cardiac tissue of mice with $I / R$ injury (Figure $3 c$ ). The enhancement in the miR-652-3p level by delivering miR-652-3p mimics remarkably reduced MTP18 expression in cardiac tissue with I/R injury (Figure 3d, upper panel), which was accompanied by a reduction in fragmented mitochondria (Figure $3 \mathrm{~d}$, lower panel) and apoptotic cells (Figures $3 e$ and f). In addition, the examination of the $\mathrm{Ml}$ area showed that the administration of miR-652-3p mimics significantly reduced the size of the infarction induced by $\mathrm{I} / \mathrm{R}$ injury (Figure $3 \mathrm{~g}$ ). The echocardiography results indicate that miR-652-3p mimics improved I/R injury-induced dysregulation in the heart (Supplementary Figures 2c-f).

To verify the connection between miR-652-3p and MTP18 in regulating mitochondrial fission and apoptosis, target protector technology was used. The target protector disrupts the specific interactions between miRNA and mRNA pairs, if they have a direct interaction. In this background, we generated an MTP18 target protector and observed its impact on the binding of miR-652-3p with MTP18 and its effect on mitochondrial fission and apoptosis. As expected, miR-652-3p did not inhibit mitochondrial fission and apoptosis in the presence of the MTP18 target protector (Supplementary Figure $3 a$ and Supplementary Figure 3b), which indicates that miR-652-3p directly interacts with MTP18. Taken together, these results confirm that miR-652-3p directly targets MTP18 to inhibit mitochondrial fission and apoptosis.

CircRNA regulates miR-652-3p expression in vivo. Our experimental results show that the expression of miR-652-3p is suppressed in cardiomyocytes under $A / R$ stress as well as in cardiac tissues with $\mathrm{I} / \mathrm{R}$ injury. This indicates that the upregulation of some factors under the above-said circumstances might negatively regulate the expression of miR-652-3p. Emerging research findings have revealed that different classes of noncoding RNAs can interact with each other and this interaction regulates their expression and/or activity. CircRNA is a class of noncoding RNAs that can act as competing endogenous sponges to regulate the expression of miRNAs. ${ }^{1,2}$ In this background, we tested whether circRNAs involved in regulating miR-652-3p 
a

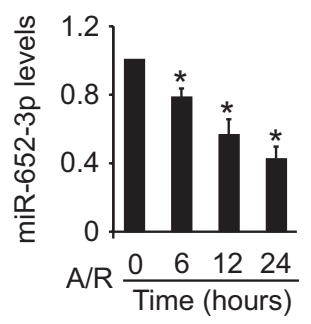

C

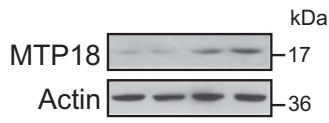

anta-652-3p (h) $0-1224$

anta-NC(h) - 24 - -

e
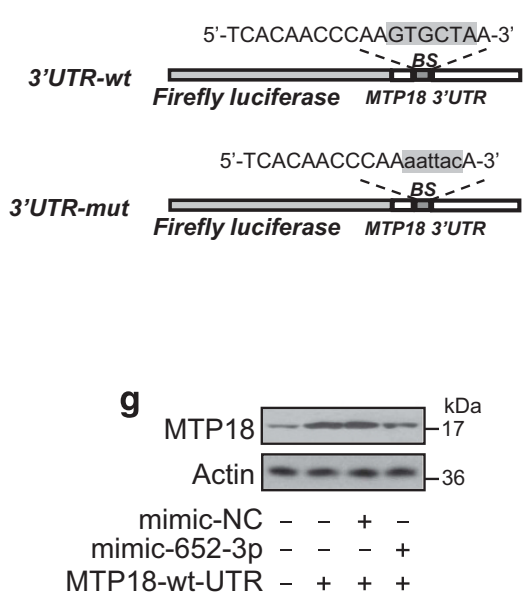

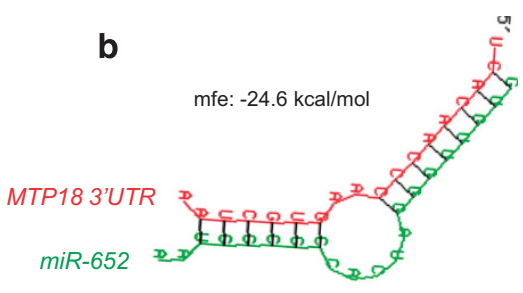

d

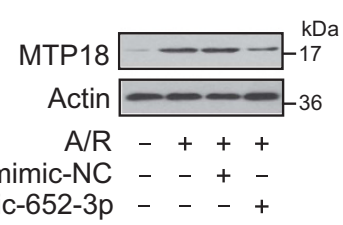

mimic-652-3p - - + +

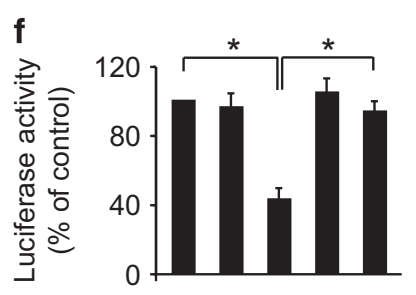

MTP18-3'UTR-wt +++-

MTP18-3'UTR-mut $-{ }_{-}++$

mimic-NC -+-+ mimic-652-3p - -+++

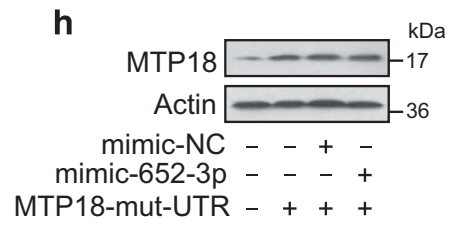

Figure 2 MiR-652-3p participates in the regulation of MTP18 expression. (a) miR-652-3p levels were analyzed by $q R T-P C R .{ }^{*} P<0.05$ versus control. (b) Analysis of MTP18 3'-UTR binding site for miR-652-3p. (c) Knockdown of miR-652-3p induces elevated MTP18 levels. Cardiomyocytes were transfected with an antagomir for miR-652-3p (anta652-3p) or a negative control (anta-NC). The expression of MTP18 was detected by immunoblot. (d) miR-652-3p attenuates A/R-induced MTP18 upregulation. Cardiomyocytes were transfected with a miR-652-3p mimic (mimic-652-3p) or its negative control (mimic-NC), and then treated with A/R. MTP18 levels were detected by immunoblot. (e) The miR-652-3p binding site was mutated in the MTP18 $3^{\prime}$-UTR. (f) miR-652-3p inhibits the luciferase activity of MTP18 with wild-type $3^{\prime}$-UTR. HEK293 cells were transfected with MTP18 with a wild-type $3^{\prime}$-UTR or mutated $3^{\prime}$-UTR as well as with a miR-652-3p mimic or its negative control. The cells were harvested and luciferase activity was measured. ${ }^{*} P<0.05$. ( $\left(\mathbf{g}\right.$ and $\mathbf{~}$ ) miR-652-3p inhibits the expression of MTP18 with wild-type $3^{\prime}-$ UTR. Cardiomyocytes were infected with adenoviral MTP18 with a wild-type $3^{\prime}-$ UTR or a mutated $3^{\prime}-$ UTR, and then were transfected with miR-652-3p mimic. The expression of MTP18 was assayed by immunoblot

expression under oxygen-deficient conditions such as anaerobic conditions and ischemia.

First, we randomly screened circRNAs from a recently published circRNA online database. ${ }^{2}$ Then, we evaluated the expression of these predicted circRNA in the mouse heart using divergent primers, and the quantitative reverse transcription-PCR (qRT-PCR) results showed that some circRNAs were expressed in the heart (Figure 4a). To determine the circRNA(s) responsible for the A/R- or I/Rinduced suppression of miR-652-3p and upregulation of mitochondrial fission and apoptosis, we assessed the expression pattern of different circRNAs in response to $A / R$ or I/R treatment. We observed a substantial increase in $\mathrm{mm9-}$ circ-016597 (Figures 4b and c), which we named MFACR. Further, we confirmed the circular nature of MFACR by using the RNase R digestion method (Figure 4d).
To test whether MFACR can bind miR-652-3p, we analyzed the complementarity of the MFACR sequence with miR-652-3p using the RNAhybrid bioinformatics program. We found that MFACR contains 15 binding sites on miR-652-3p (Figure 4e, Supplementary Figure 4 and Supplementary Figure 5). To confirm the interaction of MFACR with miR-652-3p, we carried out a RNA pull-down assay using biotin-coupled miR-652-3p mimics (Bio-652-3p-wt) and its mutant (Bio-652-3p-mut) (Figure $4 \mathrm{e}$, lower panel). In cardiomyocytes transfected with biotinylated wild-type miR-652-3p, a great amount of MFACR was captured with miR-652-3p, while a remarkable decline in the level of MFACR was observed in cells with mutated miR-652-3p, which might be due to the disturbance of the base pairing between MFACR and miR-652-3p (Figure 4f).

Next, we investigated whether MFACR serves as a platform for the binding of AGO2 and miR-652-3p. For this study, we 
a

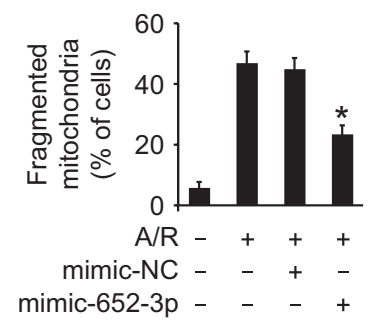

b

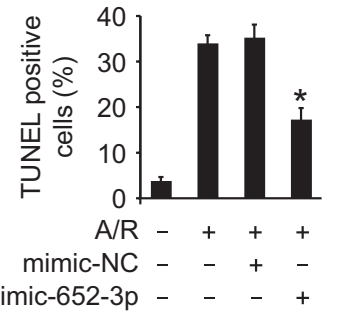

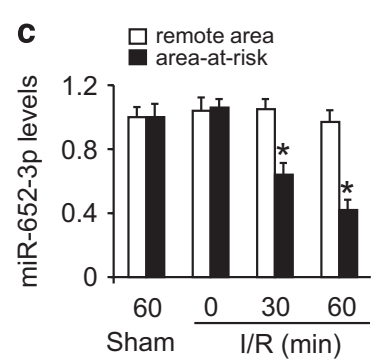
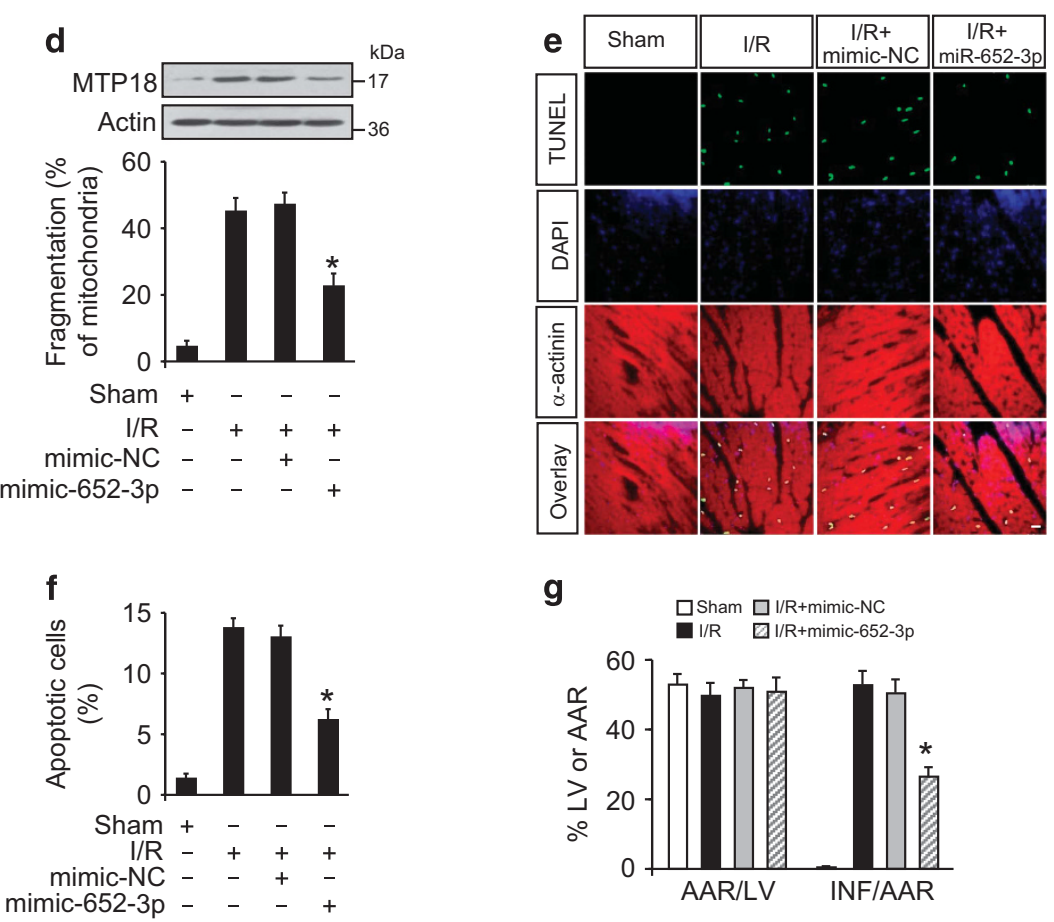

Figure 3 MiR-652-3p inhibits mitochondrial fission and apoptosis in cardiomyocytes and in vivo. (a and $\mathbf{b}$ ) miR-652-3p suppresses A/R-induced mitochondrial fission and apoptosis. Cardiomyocytes were transfected with miR-652-3p mimic or negative control (NC). After that, they were treated with A/R. Mitochondrial fission was assayed (a). Apoptosis was assayed by TUNEL (b). ${ }^{*} P<0.05$ versus A/R alone. (c) Analysis of miR-652-3p expression levels. Mice were subjected to $I / R$ at the indicated times and the expression of miR-652-3p was assayed by qRT-PCR. ${ }^{*} P<0.05$ versus sham or 0 min group. (d) miR-652-3p attenuates MTP18 expression and mitochondrial fission upon I/R injury. Adult C57BL/6 mice received miR-652-3p mimic or mimic-NC for 3 days. They were then subjected to I/R. MTP18 levels were analyzed by immunoblot (upper panel). The percentage of mitochondrial fission was calculated (lower panel). ${ }^{*} P<0.05$ versus I/R alone. (e and $\mathbf{f}$ ) miR-652-3p attenuates apoptosis upon I/R injury. Mice were treated as described in (d). Apoptosis was detected by a TUNEL assay. TUNEL-positive cardiomyocyte nuclei (apoptotic cells) are green; nuclei stained by 4',6-diamidino-2-phenylindole (DAPI) are blue. Cardiomyocytes were labeled with actinin (red). ${ }^{*} P<0.05$ versus I/R alone. (g) miR-652-3p reduces MI sizes in response to I/R. Mice were treated as described for (d), and infarct sizes were calculated. ${ }^{*} P<0.05$ versus $\mathrm{I} / \mathrm{R}$ alone

performed AGO2 immunoprecipitation in cardiomyocytes transfected with miR-652-3p or its mutant, extracted RNA from this complex, and then examined the level of MFACR using qRT-PCR. In cells transfected with native miR-652-3p, endogenous MFACR was clearly detectable in the AGO2 pull-down samples, but MFACR was not detectable in the pull-down samples from the miR-652-3p mutant-transfected cells. This indicates that miR-652-3p facilitates the AGO2 association with MFACR (Figure $4 \mathrm{~g}$ ). To validate this result, we used a reverse pull-down assay using a biotinlabeled MFACR probe and assessed the level of miR-652-3p. The northern blot analysis for miR-652-3p clearly detected the binding of MFACR to miR-652-3p (Figure 4h). Collectively, these data indicate that MFACR can directly bind to miR-652$3 p$ in vivo.
MFACR acts as a miR-652-3p sponge to regulate MTP18 expression. To study the influence of MFACR on the miR-652-3p/MTP18 axis, we used a vector-based system to overexpress MFACR. ${ }^{1}$ MFACR-ir was used as a negative control. These vectors were developed in an adenoviral system and used for further experiments. The efficiency of the constructs was analyzed by northern blotting (Figure 5a) and GRT-PCR (Figure 5b). We then analyzed whether MFACR regulates MTP18 expression and activity. The overexpression of MFACR using the adenovirus with MFACR constructs augmented the MTP18 levels in cardiomyocytes (Figure 5c), whereas knockdown of MFACR with siRNA (Supplementary Figure 6a) remarkably inhibited MFACR expression (Figure $5 \mathrm{~d}$ ). Moreover, MFACR antagonized the suppression of the MTP18 expression level (Figure 5e) and 
a

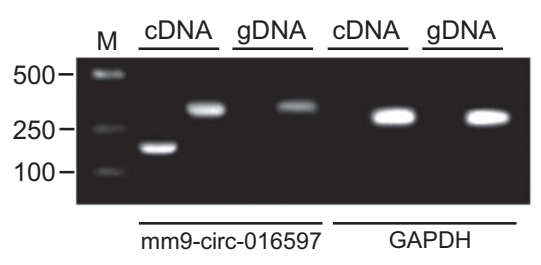

b

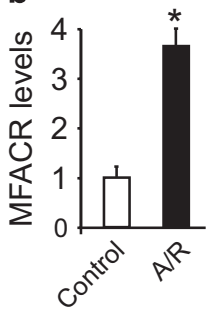

C

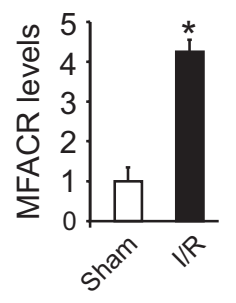

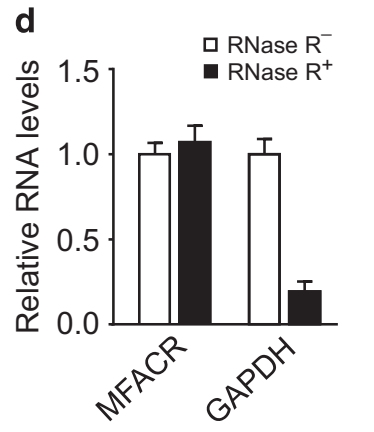

e

Splice junction

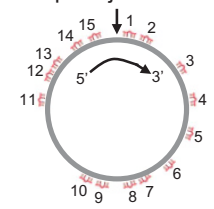

Bio-652-3p-wt

5' -AAUGGCGCCACUAGGGUUGUG-3'

Bio-652-3p-mut
5' - ACCAUAUACACUAGGGUUGUG-3'

$f$
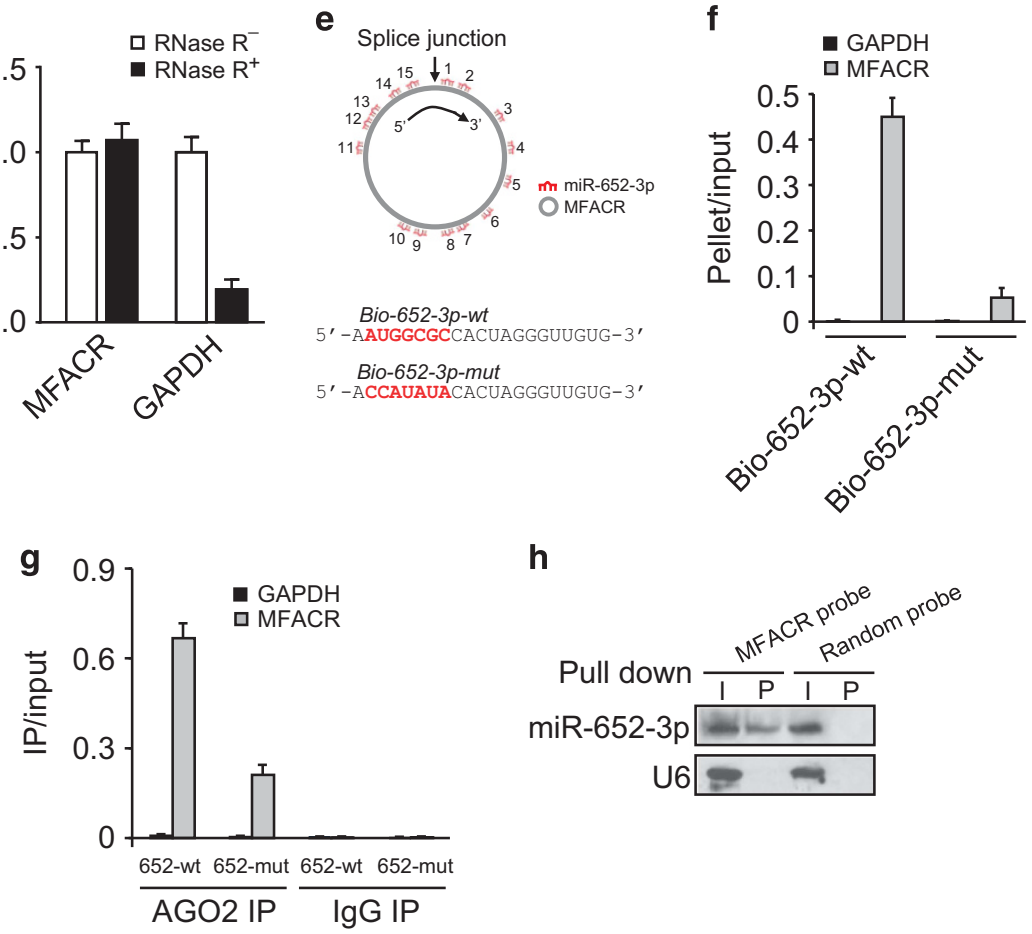

h

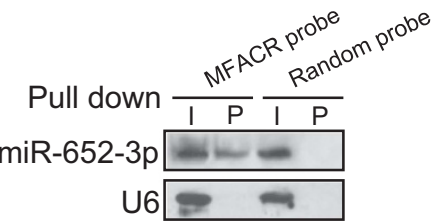

Figure 4 The circRNA MFACR binds miR-652-3p in vivo. (a) Divergent primers amplify circRNAs in cDNA but not genomic DNA (gDNA). Glyceraldehyde 3-phosphate dehydrogenase (GAPDH), linear control. (b) qRT-PCR analysis showing MFACR expression in cardiomyocytes treated with A/R. ${ }^{*} P<0.05$ versus control. (c) qRT-PCR analysis showing MFACR expression in heart samples from mice subjected to I/R or to sham treatment. ${ }^{*} P<0.05$ versus sham. (d) Real-time PCR showing resistance of MFACR to RNase R digestion. GAPDH was used as negative control. (e) MFACR RNA contains 15 sites complementary to miR-652-3p, as analyzed by the bioinformatics program RNAhybrid (upper panel). The biotin-coupled miR-652-3p mutant is shown in the lower panel. (f) The biotinylated wild-type miR-652-3p (Bio-652-3p-wt) or its mutant (Bio-652-3pmut) was transfected into cardiomyocytes. After streptavidin capture, MFACR and GAPDH mRNA levels were quantified by qRT-PCR, and the relative immunoprecipitate (IP)/ input ratios were plotted. (g) Immunoprecipitation of AGO2 from cardiomyocytes transfected with miR-652-3p or miR-652-3p mutant. MFACR and GAPDH mRNA levels were quantified by qRT-PCR, and the relative IP/input ratios were plotted. (h) miR-652-3p is associated with MFACR. miR-652-3p was pulled down by the MFACR probe or random probe. miR-652-3p levels were analyzed by northern blot. I, input; $P$, pellet

activity (Figure $5 f$ ) by miR-652-3p. The above results suggest that MFACR functions as the miR-652-3p sponge, regulating MTP18 expression and activity in cardiomyocytes.

MFACR regulates mitochondrial dynamics and apoptosis through miR-652-3p and MTP18. Next, we validated the functional role of MFACR in regulating MTP18-mediated mitochondrial fission and apoptosis in cardiomyocytes as well as in cardiac tissue. In cultured cardiomyocytes, the silencing of MFACR expression attenuated the mitochondrial fragmentation (Figure 6a) and apoptosis (Supplementary Figure $6 b$ and Figure $6 b$ ) induced by $A / R$. In mice, the knockdown of MFACR (Supplementary Figure 6c) promoted miR-652-3p expression (Supplementary Figure 6d) and decreased MTP18 protein in the heart with $\mathrm{I} / \mathrm{R}$ injury
(Supplementary Figure 6e). The knockdown of MFACR also attenuated the I/R-induced upregulation of mitochondrial fission (Figure 6c, upper panel), apoptosis (Figure 6c, lower panel), and MI size (Figure 6d). The echocardiography results revealed that the $\mathrm{I} / \mathrm{R}$ injury-induced heart dysfunction was also attenuated by knocking down MFACR expression (Supplementary Figure 6f). These data indicate that MFACR contributes to the promotion of signaling involved in mitochondrial fission and apoptotic cell death in cardiomyocytes.

To confirm that MTP18 is downstream of the MFACR/ miR-652-3p/MTP18 signaling axis, the cardiomyocytes were co-transfected with antagonists of miR-652-3p and/or MFACR. We found that the miR-652-3p antagomir abolished the effect of MFACR knockdown on MTP18 expression 
a

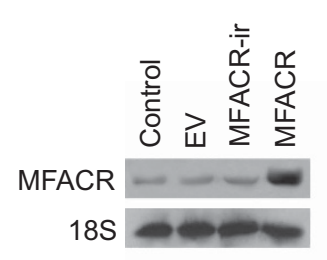

d

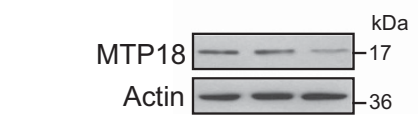

MFACR-SC - + MFACR-SiRNA - -+

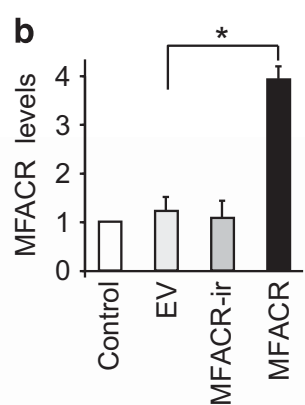

e

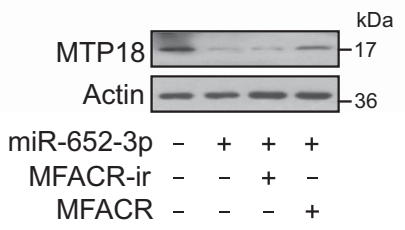

C

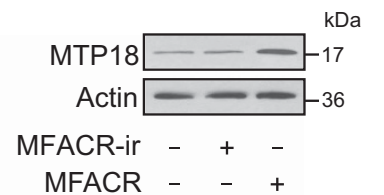

f

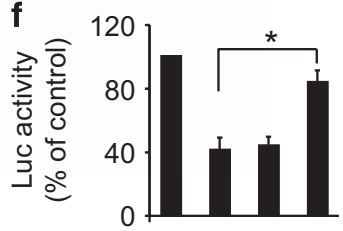

MTP18-3'UTR ++++ miR-652-3p -+++ MFACR-ir - - + -

MFACR - - -+

Figure 5 MFACR regulates MTP18 expression through miR-652-3p (a) Northern blot with $10 \mu \mathrm{g}$ of RNA from cardiomyocytes infected with adenovirus harboring empty vector (EV), MFACR-ir, or MFACR. The blot is probed against MFACR and $18 \mathrm{~S}$ ribosomal RNA (loading control). (b) Cardiomyocytes were treated as described above, and MFACR levels were analyzed by qRT-PCR. (c) Enforced expression of MFACR induces an increase in MTP18 levels. Cardiomyocytes were infected with adenoviral MFACR or MFACR-ir, and MTP18 levels were analyzed by immunoblot. (d) Knockdown of MFACR reduces the expression levels of MTP18. Cardiomyocytes were infected with adenoviral MFACR-siRNA or MFACR-sc; MTP18 levels were analyzed by immunoblot $48 \mathrm{~h}$ after infection. (e) MFACR counteracts the inhibitory effect of miR-652-3p on MTP18 expression. Cardiomyocytes were infected with adenoviral miR-652-3p, MFACR, or MFACR-ir. MTP18 levels were analyzed by immunoblot. (f) MFACR counteracts the inhibitory effect of miR-652-3p on MTP18 activity. Cardiomyocytes were infected with adenoviral miR-652-3p, MFACR, or MFACR-ir, and then transfected with an MTP18 $3^{\prime}-$ UTR luciferase construct. Luciferase activity was analyzed

(Figure 6e, upper panel), mitochondrial fission (Figure 6e, lower panel), and apoptosis (Figure 6f) in cardiomyocytes under A/R. In summary, these data reveal that MFACR, miR-652-3p, and MTP18 together forms an axis that participates in mediating mitochondrial dynamics and apoptosis in the heart.

\section{Discussion}

Noncoding RNAs have a crucial role in regulating fundamental signaling molecules associated with cardiomyocyte function as well as various cardiac diseases. The upregulation of certain miRNAs is key to the regulation of the development and progression of cardiac injury. Recent studies have shown that a class of ncRNAs called circRNAs act as an endogenous sponge and interact with miRNAs. This interaction has a great impact on the expression of miRNA target genes. For example, the human circRNA CDR1as binds to miR-7 in neuronal tissues and separates miR-7 from its target sites. ${ }^{2}$ Similarly, the circRNA Sry serves as a sponge for miR-138 and inhibits its functions. ${ }^{1,11}$ In the present study, we demonstrate that MFACR, a circRNA, positively modulates the apoptotic pathway in cardiomyocytes by targeting the miR-652-3pMTP18 signaling axis. Our study also supports the sponge activity of circRNA, which is a general characteristic of this class of ncRNA. This is the first study to illustrate that oxygen deprivation induces the upregulation of MFACR, which acts as a sponge for miR-652-3p to suppress its level. This suppression of miR-652-3p favors the upregulation of the MTP18 protein, which is a positive regulator of mitochondrial fission and apoptosis in cardiomyocytes.

In mammalian cells, a large number of circRNAs are transcribed and most of them are derived from splicing in reverse order across exons. ${ }^{19}$ For example, the Sry gene generates linear and circular transcripts in the sense direction, whereas the circRNA CDR1as is antisense to the CDR1 (cerebella degeneration-related protein 1) transcript. Our circRNA MFACR is derived from the splicing of exon 5 of the SET and MYND domain-containing 4 (Smyd4) gene in the sense direction. However, a detailed further study is required to address the mechanism of the generation and formation of MFACR.

MTP18 is a nuclear gene that codes for a mitochondrial protein capable of inducing the mitochondrial fission process. MTP18 has been proven to control mitochondrial fission in many cell types. ${ }^{12}$ However, its influence on the pathogenesis of ischemic heart disease remains largely unknown. In this study, we found that MTP18 is involved in mitochondrial fission, apoptotic cardiomyocyte death, and MI. This is the first study to report that MTP18 is a downstream target of a small ncRNA (miR-652-3p), which can inhibit the function of MTP18 by binding to the $3^{\prime}$-UTR of the MTP18 mRNA.

MFACR and miR-652-3p participate in the regulation of mitochondrial fission, cardiomyocyte apoptosis, and MI induced by I/R injury. The apoptotic cell death of cardiomyocytes caused by a low oxygen level is tightly controlled by a complex network of signaling pathways. We found that an anaerobic condition triggers the accumulation of fragmented 
a
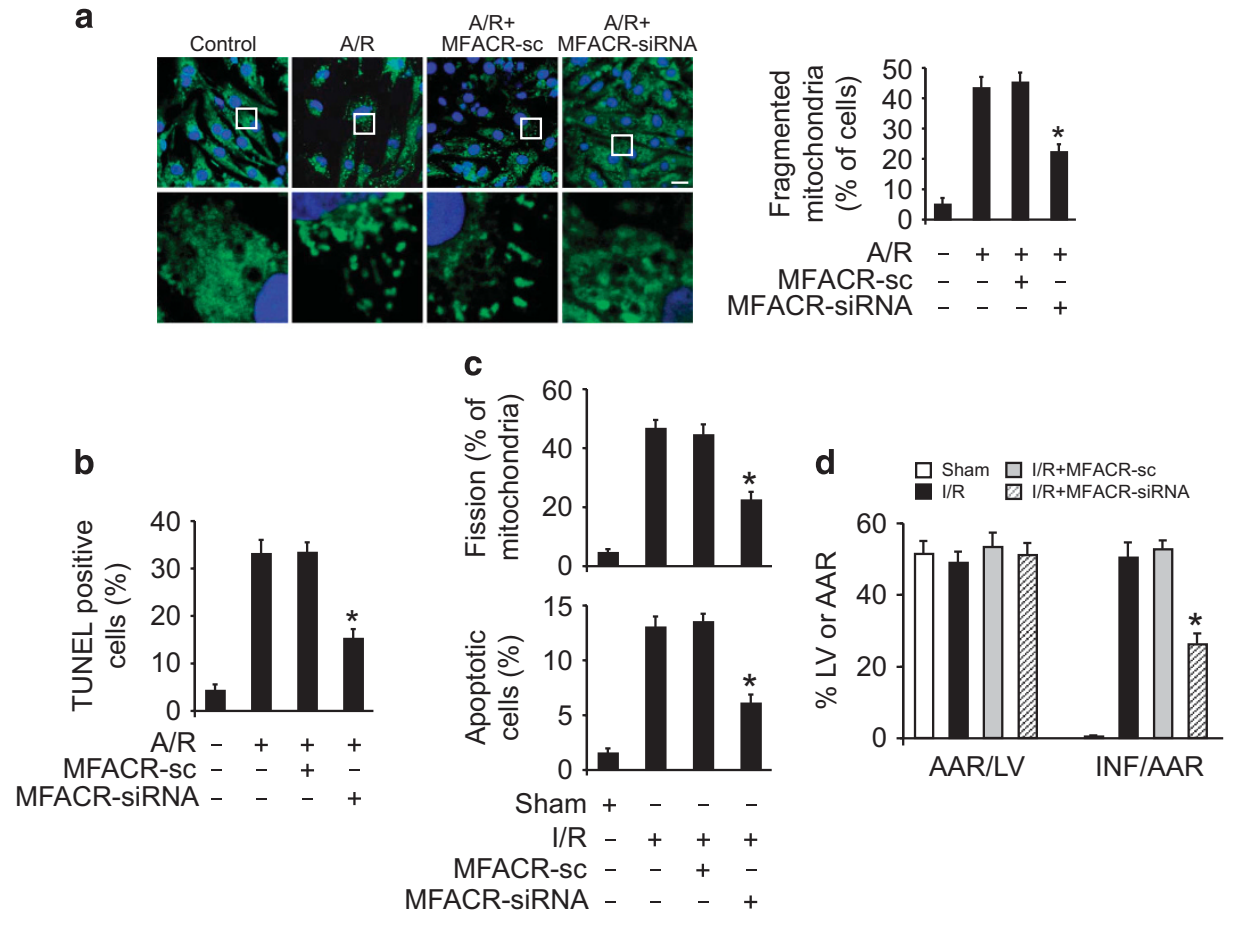

e
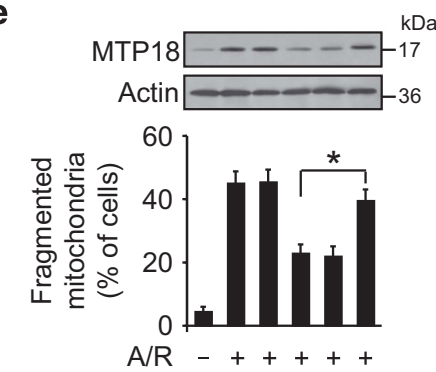

MFACR-SC $-+_{-}+$

MFACR-siRNA --++++

anta-NC $\quad \ldots+\ldots+$

anta-652-3p - - - - +

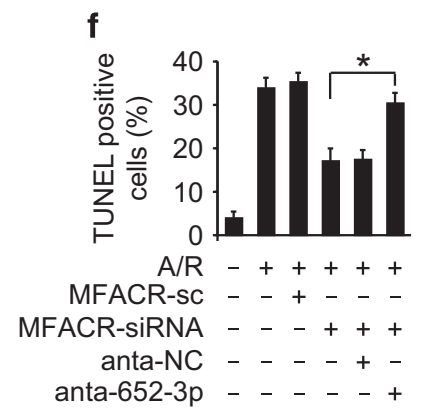

Figure 6 MFACR regulates mitochondrial fission and apoptosis by miR-652-3p and MTP18. (a) Knockdown of MFACR suppresses A/R-induced mitochondrial fission. Cardiomyocytes were infected with adenoviruses harboring MFACR-siRNA or its scrambled form and then treated with A/R. Mitochondria were stained by MitoTracker Red and nuclei were visualized by 4',6-diamidino-2-phenylindole (DAPI) (left panel). Bar $=20 \mu \mathrm{m}$. The cells with fragmented mitochondria were counted (right panel). ${ }^{*} P<0.05$ versus A/R alone. (b) Knockdown of MFACR reduces A/R-induced apoptosis. Cardiomyocytes were infected with adenoviruses harboring MFACR-siRNA or its scrambled form and then subjected to A/R. TUNEL was used to analyze apoptotic cells. ${ }^{*} P<0.05$ versus $A / R$ alone. (c) Knockdown of MFACR attenuates I/R-induced mitochondrial fission and apoptosis. After intracoronary delivery of adenoviruses harboring MFACR-siRNA or the scrambled form, mice were subjected to $45 \mathrm{~min}$ ischemia, followed by $3 \mathrm{~h}$ reperfusion. Mitochondrial fission was analyzed (upper panel). A TUNEL assay was used to detect apoptotic cells (lower panel). ${ }^{*} P<0.05$ versus I/R alone. (d) Knockdown of MFACR decreases MI sizes in response to I/R. Mice were treated as described for (c), and infarct sizes were calculated. ${ }^{*} P<0.05$ versus I/R alone. (e and f) Knockdown of miR-652-3p attenuates the inhibitory effect of MFACR knockdown on mitochondrial fission and apoptosis. Cardiomyocytes were infected with adenoviral MFACR-siRNA or MFACR-sc, transfected with the miR-652-3p antagomir (anta-652-3p) or the control (anta-NC), and then exposed to A/R. MTP18 expression was analyzed by immunoblot (e, upper panel). Mitochondrial fission (e, lower panel) and apoptosis (f) were analyzed. ${ }^{*} P<0.05$

mitochondria and consequently leads to apoptotic cell death by a mechanism of MFACR-dependent inhibition of miR-652$3 p$ and uninterrupted expression of the MTP18 protein. Although this study shows the function of MFACR in regulating cardiomyocyte cell death and pathogenesis of ischemic injury, the molecular events responsible for the generation and upregulation of MFACR in cardiomyocytes remains unknown. Our data reveal that MFACR potentially regulates miR-652-3p. Given that each ncRNA has binding sites for multiple targets, we cannot exclude the possibility of the direct/indirect involvement of other circRNAs or other classes of ncRNAs in controlling the expression of miR-652-3p.

In summary, this study demonstrates the regulatory mechanism of MTP18 and its pathogenic role in ischemic heart diseases. Our study identified for the first time that circRNA MFACR, miR-652-3p, and MTP18 together constitute a signaling pathway that regulates cardiomyocyte mitochondrial fission and apoptosis. This sheds new light on the understanding of the molecular events associated with mitochondrial fission and unravels the complex molecular mechanism of 
apoptotic cell death triggered by abnormal mitochondrial fission in cardiomyocytes. Given that a variety of stimuli can trigger cardiomyocyte apoptosis, including hydrogen peroxide, ${ }^{20}$ anoxia, ${ }^{21}$ angiotensin II, ${ }^{22}$ and staurosporine, ${ }^{23}$ it would be interesting to know if the MFACR-miR-652-3p axis could also take part in cardiomyocyte cell death under those circumstances. By addressing this question in further studies as well as by delineating the upstream regulators of MFACR, the signaling axis of MFACR/miR-652-3p/MTP18 could be a new efficient and potential target for the treatment and management of heart diseases.

\section{Materials and Methods}

Cardiomyocyte culture and treatment. Cardiomyocytes were isolated from 1- to 2-day-old mice as described. ${ }^{24}$ The cells were diluted to $1 \times 10^{6} \mathrm{cell} / \mathrm{s} / \mathrm{ml}$, plated onto different culture dishes coated with $10 \mu \mathrm{g} / \mathrm{ml}$ laminin, and treated according to the specific experimental requirements. A/R was performed as described. ${ }^{25}$ Briefly, cells were placed in an anoxic chamber with a water-saturated atmosphere composed of $5 \% \mathrm{CO}_{2}$ and $95 \% \mathrm{~N}_{2}$. After $2 \mathrm{~h}$ anoxia, the cells were subjected to reoxygenation $\left(95 \% \mathrm{O}_{2}\right.$ and $\left.5 \% \mathrm{CO}_{2}\right)$ for $12 \mathrm{~h}$.

Apoptosis assays. Apoptosis was determined by TUNEL using a kit from Roche Applied Science (Mannheim, Germany). The detection procedures were in accordance with the kit instructions. Caspase-3 was determined by analyzing its activity using an Apo-ONE Homogeneous Caspase-3/7 Assay Kit from Promega (Madison, WI, USA) according to the manufacturer's protocol.

Adenoviral constructions and infection. The circRNA MFACR vector was synthesized as described. ${ }^{1,26}$ We inserted the MFACR exon along with the endogenous flanking sequence ( $1 \mathrm{~kb}$ upstream) into pcDNA3.1, and then we copied part of the upstream flanking sequence and inserted it in an inverted orientation downstream. MFACR-ir without the downstream reverse sequence was used as a negative control. All vectors were finally cloned into the Adeno-X Expression System (Clontech, Otsu, Japan) according to the manufacturer's instructions. The mouse MTP18 was cloned by PCR using mouse cDNA as the template. The adenovirus harboring MTP18 was constructed using the Adeno-X expression system (Clontech). The mouse MTP18 RNA interference (siRNA) target sequence was 5'GGCCGATGCCATAGACAAA-3'. A scramble form was used as a control: 5'GACTGATCGAAGCAGCACA-3'. The MFACR RNA interference target sequence was 5'-GTCACACAGGGCATGTCTT-3'. A scramble form was used as a control: 5'GACTTGACGCTCGATCGCA-3'. The adenoviruses harboring siRNA or their scrambled forms were constructed using the pSilencer Adeno 1.0-CMV System (Ambion, Grandlsland, NY, USA) according to the instructions provided in the kit. All constructs were amplified in HEK293 cells. Adenoviral infection of cardiomyocytes was performed as described previously. ${ }^{24}$

Transfection of the antagomir. The chemically modified antagomir complementary to miR-652-3p designed to inhibit endogenous miR-652-3p expression and the antagomir negative control (antagomir-NC) were obtained from GenePharma Co. Ltd (Shanghai, China). The miR-652-3p antagomir sequence was 5'-CACAACCCUAGUGGCGCCAUU-3'. The antagomir-NC sequence was $5^{\prime}$ CAGUACUUUUGUGUAGUACAA- $3^{\prime}$. Cells were transfected with the antagomirs or the antagomir-NC using Lipofectamine 2000 (Invitrogen, Carlsbad, CA, USA) according to the manufacturer's instructions.

Immunoblot. Immunoblots were performed as described previously. ${ }^{27}$ Briefly, the cells were lysed for $1 \mathrm{~h}$ at $4{ }^{\circ} \mathrm{C}$ in a lysis buffer $(20 \mathrm{mM}$ Tris $(\mathrm{pH} 7.5), 2 \mathrm{mM}$ EDTA, $3 \mathrm{mM}$ EGTA, $2 \mathrm{mM}$ dithiothreitol (DTT), $250 \mathrm{mM}$ sucrose, $0.1 \mathrm{mM}$ phenylmethylsulfonyl fluoride, $1 \%$ Triton X-100) containing a protease inhibitor cocktail. The samples were subjected to $12 \%$ SDS-PAGE and transferred to nitrocellulose membranes. Equal protein loading was controlled by Ponceau Red staining of membranes. Blots were probed using primary antibodies. The antiMTP18 and anti-actin were from Abcam (Grandlsland, NY, USA). After washing four times with PBS, the appropriate horseradish peroxidase-conjugated secondary antibodies were added. Antigen-antibody complexes were visualized by enhanced chemiluminescence.
Quantitative reverse transcription-PCR. Stem-loop qRT-PCR for mature miRNAs was performed as described ${ }^{28}$ on a CFX96 Real-Time PCR Detection System (Bio-Rad, Hercules, CA, USA). Total RNA was extracted using the Trizol reagent. After DNAse I (Takara, Tokyo, Japan) treatment, RNA was reverse transcribed with reverse transcriptase (ReverTra Ace; Toyobo, Osaka, Japan). The expression of the indicated miRNAs was normalized to that of U6. The sequences of U6 primers were forward: $5^{\prime}$-GCTTCGGCAGCACATATACTAA- $3^{\prime}$ and reverse: $5^{\prime}$ AACGCTTCACGAATTTGCGT-3'. Divergent primers were designed for MFACR. The sequences of MFACR primers were forward: $5^{\prime}$-TCTTACGCACCATCATCTTG $-3^{\prime}$ and reverse: $5^{\prime}$-GTGTGCAGATTTGACTGTTT- $3^{\prime}$. The specificity of the PCR amplification was confirmed by agarose gel electrophoresis.

Preparations of the luciferase construct of MTP18 3 '-UTR and luciferase activity assay. MTP18 $3^{\prime}$-UTR was amplified by PCR. The forward primer was $5^{\prime}-$ TTCACATTGATGGGAGCTTA-3' and the reverse primer was 5'-GTTTTATTAGGTTAGCACTTG-3'. To produce the mutated $3^{\prime}$-UTR, mutations were generated using QuikChange II XL Site-Directed Mutagenesis Kit (Stratagene, La Jolla, CA, USA). The constructs were verified by sequencing. Wild-type and mutated $3^{\prime}$-UTRs were subcloned into the pGL3 vector (Promega) immediately downstream of the stop codon of the luciferase gene. Luciferase activity assay was performed using the Dual-Luciferase Reporter Assay System (Promega) according to the manufacturer's instructions.

Northern blot analysis. Northern blot was performed as we and others have described. ${ }^{29-31}$ In brief, RNA samples were electrophoresed through a $15 \%$ polyacrylamide-urea gel and transferred to positively charged nylon membranes (Millipore, Billerica, MA, USA) followed by crosslinking through UV irradiation. The membranes were subjected to hybridization with 100 pmol $3^{\prime}$-digoxigenin (DIG)labeled probe for miR-652-3p overnight at $42^{\circ} \mathrm{C}$. The miR-652-3p probe was labeled with DIG using a $3^{\prime}$-End DIG Labeling Kit (Roche Applied Science). The detection was performed using a DIG Luminescent Detection Kit (Mylab, Beijing, China) according to the manufacturer's instructions. The probe sequence for miR-652-3p was 5'-CACAACCCTAGTGGCGCCATT-3'. A DIG-labeled U6 probe was used as an internal control, and its sequence was $5^{\prime}$-TGGAACGCT TCACGAATTTG-3'.

AGO2 immunoprecipitation. miR-652-3p and miR-652-3p mutant were transfected into cardiomyocytes. Forty-eight hours after transfection, AGO2 immunoprecipitation was performed using an AGO2-specific antibody, and an IgG antibody was used as negative control. In brief, cells were lysed in $150 \mathrm{mM} \mathrm{KCl}$, $25 \mathrm{mM}$ Tris- $\mathrm{HCl}, \mathrm{pH} 7.4,5 \mathrm{mM}$ EDTA, 0.5\% Triton X-100, and $5 \mathrm{mM}$ DTT supplemented with Ribolock (Fermentas MBI, Pittsburgh, PA, USA) and proteinase inhibitor cocktail (Roche Applied Science). The lysate was mixed with antibodycoupled Sepharose beads and left under rotation for $4 \mathrm{~h}$ at $4{ }^{\circ} \mathrm{C}$. Beads were subsequently washed six times in lysis buffer and the RNA was extracted using Trizol reagent (Invitrogen).

Mitochondrial staining. Mitochondrial staining was carried as we and others have described with modifications. ${ }^{32,33}$ Briefly, cells were plated onto coverslips coated with $0.01 \%$ poly-L-lysine. After treatment, they were stained for 20 min with $0.02 \mu \mathrm{M}$ MitoTracker Red CMXRos (Molecular Probes, Life Technologies Co., Grand Island, NY, USA). Mitochondria were imaged using a laser scanning confocal microscope (Zeiss LSM510 META, Oberkochen, Germany). The percentage of cells with fragmented mitochondria relative to the total number of cells is presented as the mean \pm S.E.M. of at least three independent experiments, counted by an observer blinded to the experimental conditions. Six distinct fields, each with at least 50 cells, were counted. At least 300 cells per group were counted.

Echocardiographic assessment of cardiac dimensions and function. Transthoracic echocardiography was performed on lightly anesthetized mice using a Vevo 770 high-resolution system (Visualsonics, Toronto, ON, Canada) equipped with a $40 \mathrm{MHz}$ RMV 704 scan head. Two-dimensional guided M-mode tracings were recorded in both parasternal long and short axis views at the level of the papillary muscles. Fractional shortening, left ventricular ejection fraction, left ventricular end-systolic volume and left ventricular end-diastolic volume were measured by echocardiography. All measurements were taken from more than three beats and averaged. 
Animal experiments. Male adult C57BL/6 mice ( 8 weeks old) were obtained from the Institute of Laboratory Animal Science of the Chinese Academy of Medical Sciences (Beijing, China). All experiments were performed according to the protocols approved by the Institute Animal Care Committee. The mice received intravenous injections of miR-652-3p mimic or its control at a dose of $35 \mathrm{mg} / \mathrm{kg}$ body weight in a small volume $(0.2 \mathrm{ml}$ per injection) for three consecutive days.

I/R injury was surgically induced in mice as we described previously. ${ }^{25}$ Briefly, all mice were subjected to $45 \mathrm{~min}$ ischemia, followed by $3 \mathrm{~h}$ reperfusion. The shamoperated group experienced the same procedure, except that the snare was left untied. Cardiac function in these groups of animals was evaluated by echocardiographic analysis 14 days after the surgery. Evans blue dye analysis was performed as described. ${ }^{25}$ The areas of infarction, area at risk (AAR), and nonischemic left ventricle (LV) were assessed with computer-assisted planimetry (NIH Image 1.57) by an observer blinded to the sample identity. The ratio of AAR/LV and INF/AAR were calculated as described. ${ }^{25}$

Five days before the $I / R$ operation, the mice were anesthetized to perform intracoronary delivery of adenovirus. The chest was opened and MTP18-siRNA adenoviruses $\left(2 \times 10^{10} \mathrm{MOI}\right)$ or MFACR-siRNA adenoviruses $\left(2 \times 10^{11} \mathrm{MOI}\right)$ were injected with a catheter from the apex of the LV into the aortic root, while the aorta and pulmonary arteries were cross-clamped. The clamp was maintained for $20 \mathrm{~s}$ while the heart pumped against a closed system. The chest was closed and the animal was transferred back to its cage for recovery.

Statistical analyses. Data are expressed as the mean \pm S.E.M. of at least three independent experiments. One-way analysis of variance was used for multiple comparisons. A value of $P<0.05$ was considered significant.

\section{Conflict of Interest}

The authors declare no conflict of interest.

Acknowledgements. This work was supported by the National Natural Science Foundation of China (81522005, 81470522, 31430041, and 81230005), and the Taishan Scholar Program of Shandong Province.

1. Hansen TB, Jensen TI, Clausen BH, Bramsen JB, Finsen B, Damgaard CK et al. Natural RNA circles function as efficient microrna sponges. Nature 2013; 495: 384-388.

2. Memczak S, Jens M, Elefsinioti A, Torti F, Krueger J, Rybak A et al. Circular RNAs are a large class of animal RNAs with regulatory potency. Nature 2013; 495: 333-338.

3. Rybak-Wolf A, Stottmeister C, Glazar P, Jens M, Pino N, Giusti S et al. Circular RNAs in the mammalian brain are highly abundant, conserved, and dynamically expressed. Mol Cell 2015; 58: 870-885

4. Ashwal-Fluss R, Meyer M, Pamudurti NR, Ivanov A, Bartok O, Hanan M et al. CircRNA biogenesis competes with pre-mRNA splicing. Mol Cell 2014; 56: 55-66.

5. Lu T, Cui L, Zhou Y, Zhu C, Fan D, Gong H et al. Transcriptome-wide investigation of circular RNAs in rice. RNA (New York, NY) 2015; 21: 2076-2087.

6. Koh W, Pan W, Gawad C, Fan HC, Kerchner GA, Wyss-Coray T et al. Noninvasive in vivo monitoring of tissue-specific global gene expression in humans. Proc Natl Acad Sci USA 2014; 111: 7361-7366.

7. Lukiw W. Circular RNA (circRNA) in Alzheimer's disease (AD). Front Genet 2013; 4: 307.

8. Bachmayr-Heyda A, Reiner AT, Auer K, Sukhbaatar N, Aust S, Bachleitner-Hofmann T et al. Correlation of circular RNA abundance with proliferation - exemplified with colorectal and ovarian cancer, idiopathic lung fibrosis, and normal human tissues. Scientific Rep 2015; 5: 8057

9. Qu S, Song W, Yang X, Wang J, Zhang R, Zhang Z et al. Microarray expression profile of circular rnas in human pancreatic ductal adenocarcinoma. Genomics Data 2015; 5: 385-387.

10. Hansen TB, Kjems J, Damgaard CK. Circular RNA and mir-7 in cancer. Cancer Res 2013; 73: $5609-5612$.
11. Capel B, Swain A, Nicolis S, Hacker A, Walter M, Koopman P et al. Circular transcripts of the testis-determining gene sry in adult mouse testis. Cell 1993; 73: 1019-1030.

12. Tondera D, Czauderna F, Paulick K, Schwarzer R, Kaufmann J, Santel A. The mitochondrial protein mtp18 contributes to mitochondrial fission in mammalian cells. J Cell Sci 2005; 118 : 3049-3059.

13. Tondera D, Santel A, Schwarzer R, Dames S, Giese K, Klippel A et al. Knockdown of mtp18, a novel phosphatidylinositol 3-kinase-dependent protein, affects mitochondrial morphology and induces apoptosis. J Biol Chem 2004; 279: 31544-31555.

14. Roderburg C, Mollnow T, Bongaerts B, Elfimova N, Vargas Cardenas D, Berger $\mathrm{K}$ et al. Micro-rna profiling in human serum reveals compartment-specific roles of mir-571 and mir652 in liver cirrhosis. PLOS ONE 2012; 7: e32999.

15. Andersen M, Grauslund M, Ravn J, Sorensen JB, Andersen CB, Santoni-Rugiu E. Diagnostic potential of mir-126, mir-143, mir-145, and mir-652 in malignant pleural mesothelioma. J Mol Diagn 2014; 16: 418-430.

16. Gilicze AB, Wiener Z, Toth $\mathrm{S}$, Buzas $\mathrm{E}$, Pallinger $\mathrm{E}$, Falcone $\mathrm{FH}$ et al. Myeloid-derived micrornas, mir-223, mir27a, and mir-652, are dominant players in myeloid regulation. BioMed Res Int 2014; 2014: 870267

17. Deng S, Li X, Niu Y, Zhu S, Jin Y, Deng S et al. Mir-652 inhibits acidic microenvironmentinduced epithelial-mesenchymal transition of pancreatic cancer cells by targeting zeb1. Oncotarget 2015; 6: 39661-39675.

18. Yang W, Zhou C, Luo M, Shi X, Li Y, Sun Z et al. Mir-652-3p is upregulated in non-small cell lung cancer and promotes proliferation and metastasis by directly targeting Igl1. Oncotarget 2016; 7: 16703-16715.

19. Wang $Y$, Wang Z. Efficient backsplicing produces translatable circular mRNAs. RNA (New York, NY) 2015; 21: 172-179.

20. Barlaka E, Gorbe A, Gaspar R, Paloczi J, Ferdinandy P, Lazou A. Activation of pparbeta/ delta protects cardiac myocytes from oxidative stress-induced apoptosis by suppressing generation of reactive oxygen/nitrogen species and expression of matrix metalloproteinases. Pharmacol Res 2015; 95-96: 102-110.

21. Hu J, Chu Z, Han J, Zhang Q, Zhang D, Dang Y et al. Phosphorylation-dependent mitochondrial translocation of map4 is an early step in hypoxia-induced apoptosis in cardiomyocytes. Cell Death Dis 2014; 5: e1424.

22. Zhao H, Qi G, Han Y, Shen X, Yao F, Xuan C et al. 20-Hydroxyeicosatetraenoic acid is a key mediator of angiotensin II-induced apoptosis in cardiac myocytes. $J$ Cardiovasc Pharmacol 2015; 66: 86-95.

23. Liu AH, Cao YN, Liu HT, Zhang WW, Liu Y, Shi TW et al. Dids attenuates staurosporineinduced cardiomyocyte apoptosis by pi3k/akt signaling pathway: activation of eNOS/NO and inhibition of bax translocation. Cell Physiol Biochemis 2008; 22: 177-186.

24. Tan WQ, Wang K, Lv DY, Li PF. Foxo3a inhibits cardiomyocyte hypertrophy through transactivating catalase. J Biol Chem 2008; 283: 29730-29739.

25. Wang JX, Jiao JQ, Li Q, Long B, Wang K, Liu JP et al. Mir-499 regulates mitochondrial dynamics by targeting calcineurin and dynamin-related protein-1. Nat Med 2011; 17: 71-78.

26. Pasman Z, Been MD, Garcia-Blanco MA. Exon circularization in mammalian nuclear extracts. RNA (New York, NY) 1996; 2: 603-610.

27. Li PF, Li J, Muller EC, Otto A, Dietz R, von Harsdorf R. Phosphorylation by protein kinase ck2: a signaling switch for the caspase-inhibiting protein arc. Mol Cell 2002; 10: 247-258.

28. Chen C, Ridzon DA, Broomer AJ, Zhou Z, Lee DH, Nguyen JT et al. Real-time quantification of micrornas by stem-loop RT-PCR. Nucleic Acids Res 2005; 33: e179.

29. Lanza G, Ferracin M, Gafa R, Veronese A, Spizzo R, Pichiorri F et al. MRNA/microRNA gene expression profile in microsatellite unstable colorectal cancer. Mol Cancer 2007; 6: 54.

30. Lau P, Verrier JD, Nielsen JA, Johnson KR, Notterpek L, Hudson LD. Identification of dynamically regulated microRNA and mRNA networks in developing oligodendrocytes. $J$ Neurosci 2008; 28: 11720-11730.

31. Li YZ, Lu DY, Tan WQ, Wang JX, Li PF. P53 initiates apoptosis by transcriptionally targeting the anti-apoptotic protein arc. Mol Cell Biol 2008; 28: 564-574.

32. Frank S, Gaume B, Bergmann-Leitner ES, Leitner WW, Robert EG, Catez F et al. The role of dynamin-related protein 1, a mediator of mitochondrial fission, in apoptosis. Dev Cell 2001; 1 : 515-525.

33. Wang JX, Li Q, Li PF. Apoptosis repressor with caspase recruitment domain contributes to the chemotherapy resistance by abolishing mitochondrial fission mediated by dynaminrelated protein-1. Cancer Res 2009; 69: 492-500. 